\title{
Mechanical Occlusion Chemically Assisted Ablation (MOCA) for Saphenous Vein Insufficiency: A Meta-Analysis of a Randomized Trial
}

\author{
Johanes Nugroho $\left(\mathbb{D},{ }^{1,2}\right.$ Ardyan Wardhana $\mathbb{D}^{3},{ }^{3}$ and Cornelia Ghea $\mathbb{D}^{3}$ \\ ${ }^{1}$ Department of Cardiology and Vascular Medicine, Faculty of Medicine, Universitas Airlangga, Surabaya, Indonesia \\ ${ }^{2}$ Dr. Soetomo General Hospital, Surabaya, Indonesia \\ ${ }^{3}$ PILAR Research and Education, Cambridge, UK
}

Correspondence should be addressed to Johanes Nugroho; j.nugroho.eko@fk.unair.ac.id

Received 26 October 2019; Revised 24 December 2019; Accepted 13 January 2020; Published 29 January 2020

Academic Editor: Alberto Caggiati

Copyright ( 2020 Johanes Nugroho et al. This is an open access article distributed under the Creative Commons Attribution License, which permits unrestricted use, distribution, and reproduction in any medium, provided the original work is properly cited.

\begin{abstract}
Purpose. A previous meta-analysis has conducted nonrandomized trials for mechanochemical ablation (MOCA). Since mediumterm follow-up data from randomized clinical trials (RCTs) are becoming available, we chose to perform a meta-analysis of RCTs to assess the efficacy and safety of MOCA for saphenous vein insufficiency. Methods. A systematic search of all RCTs comparing the anatomical success of MOCA for saphenous vein insufficiency to thermal ablation was performed using the PubMed and Cochrane Library databases. We employed the Mantel-Haenszel random-effects meta-analysis of outcomes using RevMan 5.3. Results. Four studies (615 patients) were included in this meta-analysis. The MOCA group had $93.4 \%$ and $84.5 \%$, whereas the thermal ablation group had $95.8 \%$ and $94.8 \%$ of anatomical success rate at 1 month (short-term) and a period of more than 6 months but less than 1-year follow-up (mid-term), respectively. According to intention-to-treat analysis, there were similar anatomical successes in MOCA and thermal ablation groups at the short-term follow-up (low-quality evidence; relative risk $\left.(\mathrm{RR})=0.98(95 \% \mathrm{CI}, 0.94-1.03) ; P=0.44 ; I^{2}=53 \%\right)$. The estimated effect of MOCA on anatomical success showed a statistically significant reduction at the mid-term follow-up (moderate-quality evidence; $\mathrm{RR}=0.89$ (95\% CI, 0.84-0.95); $P=$ $\left.0.0002 ; I^{2}=0 \%\right)$. MOCA had fewer incidence of nerve injury, deep vein thrombosis, and skin burns compared to the thermal ablation procedure (low-quality evidence; $\mathrm{RR}=0.33$ (95\% CI, 0.09-1.28); $P=0.11 ; I^{2}=0 \%$ ). Conclusion. MOCA offered fewer major complications but lesser anatomical success at the period of more than 6 months but less than 1-year follow-up than thermal ablation. Trial Registration. This trial is registered with UMIN Clinical Trial Registry (UMIN ID 000036727).
\end{abstract}

\section{Introduction}

Saphenous vein insufficiency is the most common chronic venous incompetence of lower limbs. It affects more than $30 \%$ of adults [1]. The first-line treatment of venous incompetence is thermal ablation, e.g., radiofrequency ablation (RFA) and endovenous laser ablation (EVLA) [2]. However, the use of heat energy in thermal ablation was associated with complications such as nerve injury and skin burns $[3,4]$.

To avoid thermal energy complications, few novel treatments such as sclerotherapy, cyanoacrylate, and mechanochemical ablation (MOCA) have been introduced. One of MOCA device is ClariVein (Merit Medical, South Jordan,
Utah) that combines mechanical injury to the venous endothelium using a rotating wire with simultaneous delivery and dispersion of a liquid sclerosant [5]. The liquid sclerosant causes irreversible damage to the cellular membranes of the endothelium, resulting in the fibrosis of veins [6]. Another technique to produce a nontumescent sclerosantassisted ablation is by means of Flebogrif (Balton, Poland), a device that is scratching the endothelium lining by a dedicated catheter.

A previous meta-analysis has conducted nonrandomized trials for MOCA [7]. It is important to study the use of MOCA since medium-term follow-up data on randomized clinical trials are now becoming available. Therefore, we 
performed a meta-analysis of randomized trials aiming to assess the safety and efficacy of MOCA by comparing thermal ablation for saphenous vein insufficiency.

\section{Methods}

All RCTs comparing the anatomical success of MOCA for saphenous vein insufficiency (great saphenous vein (GSV), small saphenous vein (SSV), or both) to the success rate of a thermal ablation procedure were eligible for inclusion in this review. All trials comparing MOCA to treatments other than thermal ablation were excluded. A systematic search of the literature was undertaken on July 7, 2019, after receiving an approval from the Institutional Review Board. Two different databases (PubMed and Cochrane Library) were used to perform a systematic search of all the literature without language restriction. Search terms were differently spelled text words or abbreviations, such as "varicose," "saphenous incompetence," "saphenous insufficiency," "saphenous varicose veins," "saphenous reflux," "saphenous vein," "endovenous ablation," "clarivein," "MOCA," "mechanochemical ablation," "randomized," and "rct" in the title, abstract, and medical subject heading (MeSH). Reference lists of the included studies were also evaluated to identify additional relevant studies. We followed the Preferred Reporting Items for Systematic Reviews and Meta-Analyses guidelines for research reporting.

Two investigators independently screened and assessed titles and abstracts before full-text retrieval. The full papers that potentially met the inclusion/exclusion criteria were reviewed by the two authors for final inclusion. Subsequently, two investigators extracted the data, which included authors, year of publication, detailed intervention agent, veins treated (either GSVs or SSVs), postprocedural management, number of patients, primary outcomes, and adverse events. The corresponding author was contacted to obtain any incomplete data. All extracted data were recorded with a dedicated data extraction form on an Excel spreadsheet.

The primary outcome in our meta-analysis was anatomical success that was defined either as complete occlusion of the saphenous vein or at least proximal occlusion $(>5 \mathrm{~cm}$ proximally occluded with $>5 \mathrm{~cm}$ distally open) on duplex ultrasound (DUS) imaging. The primary outcome was categorized at the short-term follow-up ( 1 month) and mid-term follow-up ( $\geq 6$ months but $\leq 1$ year). Secondary outcomes were phlebitis and major adverse event rates, including nerve injury, DVT, and skin burns. Nerve injury was reported differentially in the studies as sensory disturbance, paresthesia, or numbness. Other minor complications (e.g., hematoma, lumps, and hyperpigmentation) were excluded from the analysis.

Two authors independently assessed the methodological quality of the articles using the Risk of Bias Tool (Cochrane collaboration). They employed a GRADE approach to evaluate the quality of evidence $[8,9]$. Factors assessed for the risk of bias were as follows [1]: randomization [2], adherence to intervention [3], measurement [4], missing outcome data, and reporting [5]. We evaluated the GRADE while taking into account the following factors: risk of bias, imprecision, indirectness of evidence, inconsistency in results among included studies, and reporting bias.

We conducted the meta-analysis using relative risk (RR) for anatomical success rates at short-term and mid-term follow-up periods. We employed the Mantel-Haenszel random-effects method using Review Manager (RevMan v5.3 2014). We conducted an intention-to-treat (ITT) analysis using imputation data according to event rates among completers in the separate groups [10]. Available case analysis was further performed as a sensitivity analysis. We evaluated between and within-study heterogeneity using the $I^{2}$ statistic.

\section{Results}

A total of 100 studies were identified after our initial search was completed (45 citations in PubMed and 55 in Cochrane Library, as presented in Figure 1). Six studies were further retrieved to review the full text. However, two studies were excluded because one lacked a control and one was an inabstract study $[11,12]$. Therefore, four studies were finally included in our analysis [13-16]. Attempts to contact authors to acquire unpublished data were uniformly unsuccessful. As such, only information available from the publication was used. Table 1 summarizes the clinical characteristics of the included studies.

The Cochrane risk of bias analysis is shown in Figure 2. There was a low risk of a randomization process in all included trials. Blinding to participants and the delivering clinicians was not performed in all included trials. There was no placebo included for comparison. However, there was assessor-blinding when evaluating the anatomical success using DUS imaging. One trial was stopped early because reimbursement of MOCA was suspended [13]. Participants who were not followed up ranged from $6 \%$ to $34 \%$, and there were no documented reasons for loss to follow-up. All trials reported the results from available case analyses and not ITT analysis.

Table 2 shows the quality of evidence, and the GRADE approach was used for each outcome. We did not downgrade from the risk of bias aspect because most information was from studies that were at low risk of bias. We downgraded one level for the evidence of anatomical success at the short-term follow-up because there was substantial heterogeneity. Indirectness did not appear to be an issue in all outcomes. We downgraded the evidence of major complications for the imprecision aspect because there was a wide CI that included no important effect and RR reduction greater than $25 \%$. The publication bias was assessed for all outcomes because there was asymmetry from a visual inspection of the funnel plot (Figure 3). This finding was not further evaluated by conducting Egger's test because there were less than 10 included trials.

The MOCA group had $93.4 \%$ of anatomical success rate, whereas the thermal ablation group had $95.8 \%$ at the 1-month follow-up. According to the meta-analysis using ITT as shown in Table 3, there were similar anatomical successes in the MOCA and thermal ablation groups at the 1-month follow-up ( 4 trials; $\mathrm{RR}=0.98$ (95\% CI, 0.94- 


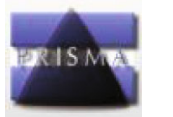

PRISMA 2009 Flow Diagram
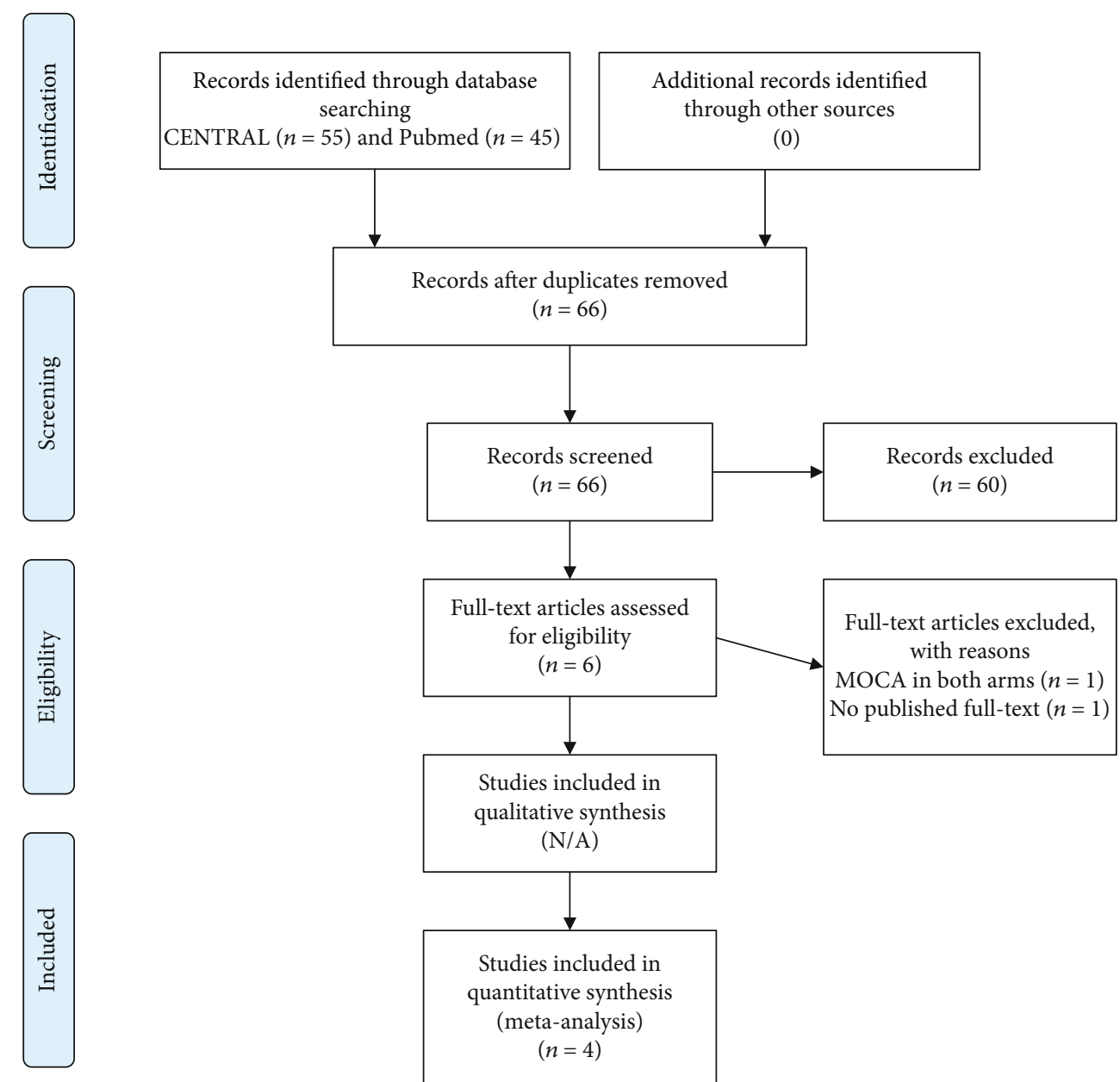

Studies included in qualitative synthesis (N/A)

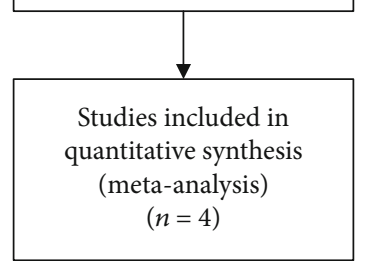

Figure 1: PRISMA flow diagram.

1.03); $P=0.44)$; however, there was substantial heterogeneity $\left(I^{2}=53 \% ; P=0.09\right)$. At the mid-term follow-up $(\geq 6$ months but $\leq 1$ year), the MOCA group had $84.5 \%$ of anatomical success rate, whereas the thermal ablation group had $94.8 \%$ (Table 4). The estimated effect of MOCA on anatomical success showed a statistically significant reduction at the mid-term follow-up ( 3 trials; $\mathrm{RR}=0.89$ (95\% CI, 0.84-0.95); $P=0.0002)$, without substantial heterogeneity $\left(I^{2}=0 \% ; P=0.52\right)$.

We performed sensitivity analysis using the available case analyses. The direction of effect did not change for anatomical success rates. MOCA did not decrease anatomical success compared to thermal ablation at the 1-month follow-up (4 trials; $\mathrm{RR}=0.98$ (95\% CI, 0.93-1.03); $P=0.46$ ); however, there was substantial heterogeneity $\left(I^{2}=56 \% ; P=0.08\right)$. The RR for anatomical success at the mid-term follow-up ( $\geq 6$ months but $\leq 1$ year) was significantly lower after treatment with MOCA than that with thermal ablation ( 3 trials;
$\mathrm{RR}=0.89$ (95\% CI, 0.84-0.96); $P=0.001)$, without substantial heterogeneity $\left(I^{2}=0 \% ; P=0.67\right)$.

Table 5 shows the details of adverse events in the four included trials. MOCA had fewer major complications compared to thermal ablation procedures (4 trials, $\mathrm{RR}=0.33$ (95\% CI, 0.09-1.28); $\left.P=0.11 ; I^{2}=0 \%\right)$. However, participants in the MOCA group had a higher risk of phlebitis than the thermal ablation group (4 trials, $\mathrm{RR}=1.39$ (95\% CI, 0.67-2.86); $\left.P=0.37 ; I^{2}=0 \%\right)$.

\section{Discussion}

We report that MOCA had a similar anatomical success rate with low-quality evidence at the 1-month follow-up, but there was a reduction of the anatomical success rate with moderate-quality evidence at the period of more than 6 months but less than 1-year follow-up compared with thermal ablation. Previous meta-analyses that included 


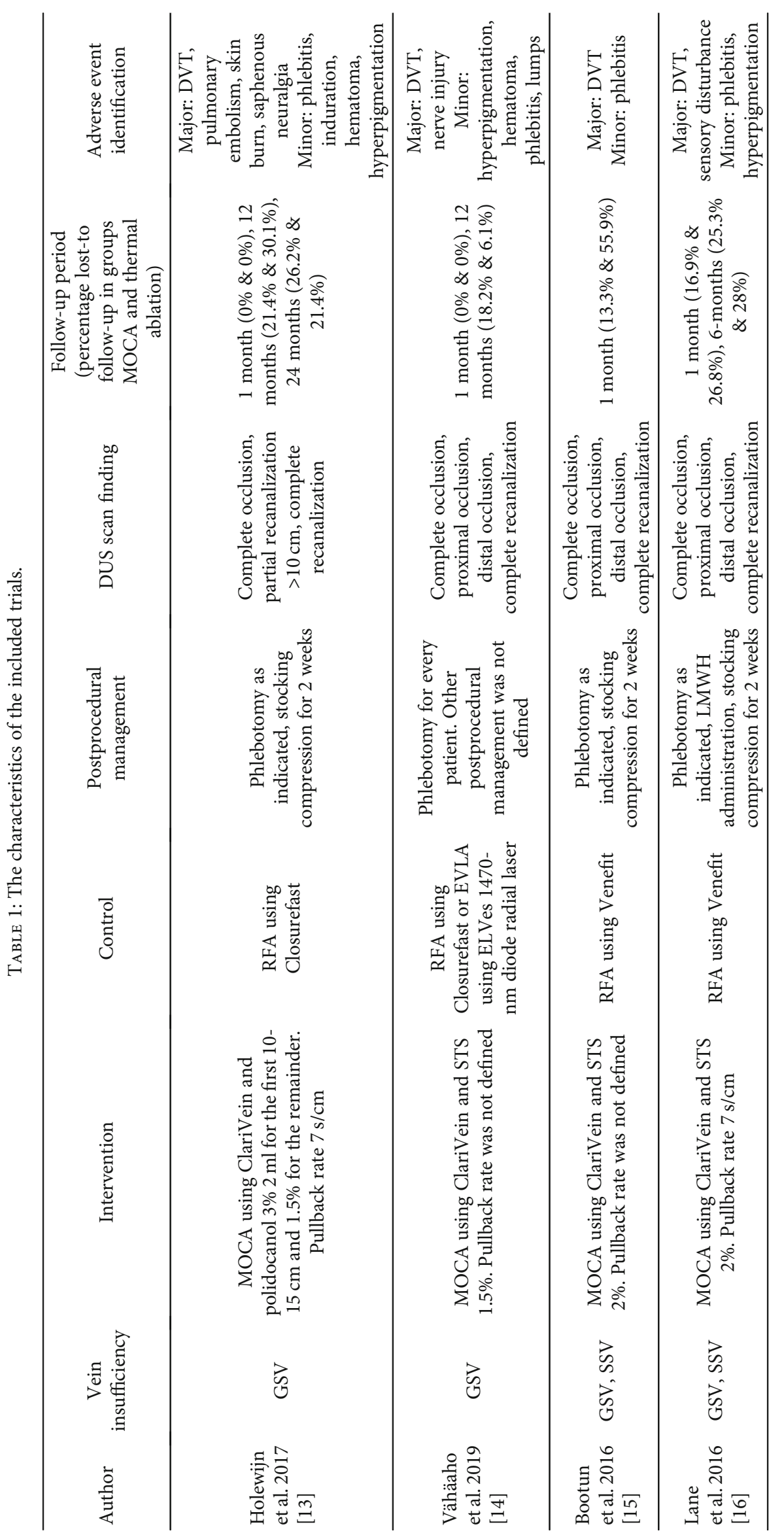




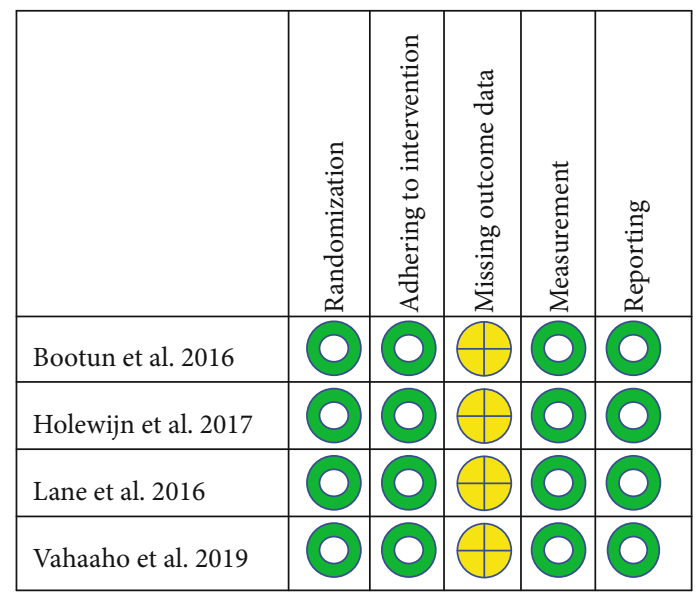

(O) = Low risk
$\theta=$ Unclear risk

FIGURE 2: Risk of bias assessment.

TABLE 2: GRADE assessment.

\begin{tabular}{|c|c|c|c|c|c|c|c|}
\hline Comparison & Outcomes & Risk of bias & Inconsistency & Indirectness & Imprecision & Publication of bias & GRADE \\
\hline \multirow{2}{*}{ Anatomical success } & Short-term & No serious & Some & No at all & No & Presence & Low \\
\hline & Mid-term & No serious & No & No at all & No & Presence & Moderate \\
\hline \multirow{2}{*}{ Complication } & Major complication & No serious & No & No at all & Some & Presence & Low \\
\hline & Phlebitis & No serious & No & No at all & No & Presence & Moderate \\
\hline
\end{tabular}

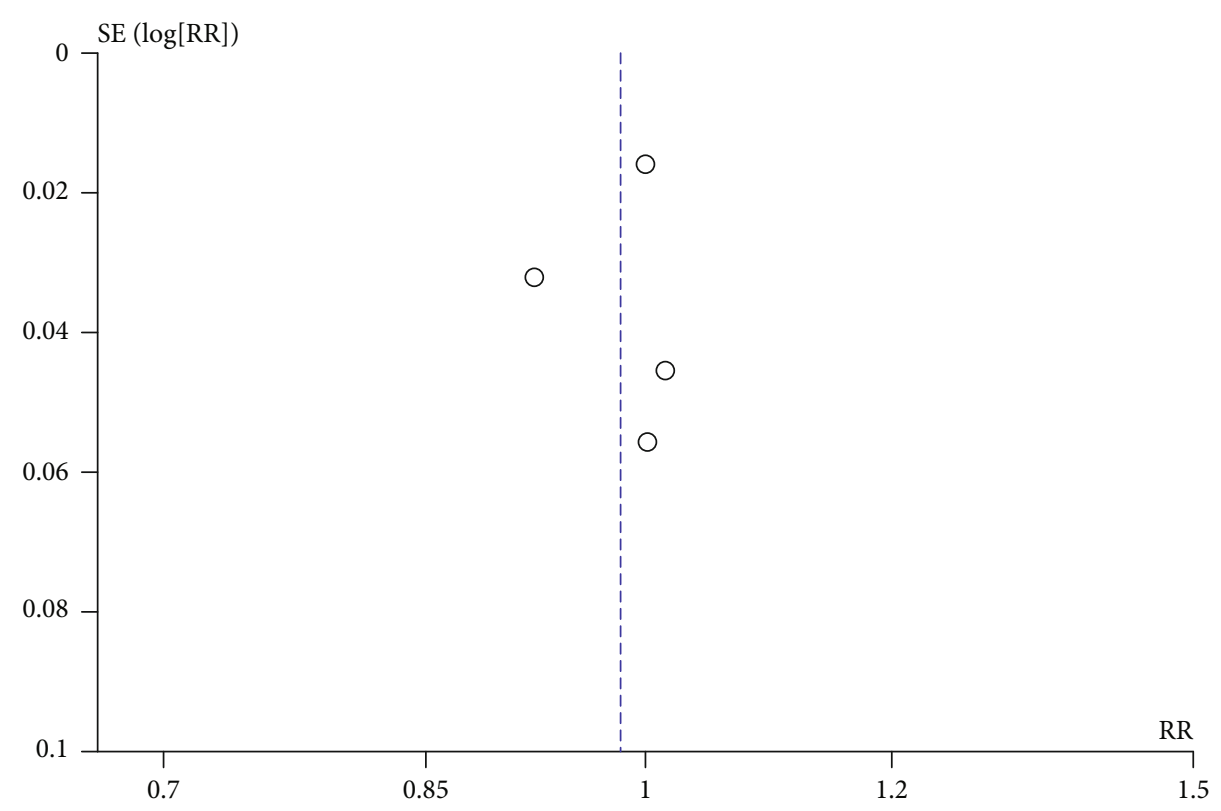

FIGURE 3: Funnel plot of comparisons: mechanochemical ablation vs thermal ablation intention-to-treat analysis, outcome: anatomical success short-term.

nonrandomized trials demonstrated that MOCA could be considered an effective treatment for saphenous vein insufficiency; however, these results were based on a pooled analysis of low-quality data [17]. In previous studies, the pooled ana- tomical success rate at the mid-term follow-up was $91 \%$, 97.1\%, and 98.5\% for MOCA, RFA, and EVLA, respectively $[7,18]$; the rates obtained in our study were lower: $84.5 \%$ for MOCA and $94.8 \%$ for thermal ablation. Blinding to the 
TABLE 3: Summary of findings.

\begin{tabular}{|c|c|c|c|c|c|}
\hline & & Studies & Participants & Effect estimate & Heterogeneity \\
\hline \multirow{2}{*}{ ITT analysis } & Anatomical success at short-term & 4 & 615 & RR $0.98[0.94,1.03] ; P=0.44$ & $P=0.09 ; I^{2}=53 \%$ \\
\hline & Anatomical success at mid-term & 3 & 496 & RR $0.89[0.84,0.95] ; P=0.0002$ & $P=0.53 ; I^{2}=0 \%$ \\
\hline \multirow[b]{2}{*}{ Available case analysis } & Anatomical success at short-term & 4 & 532 & RR $0.98[0.93,1.03] ; P=0.46$ & $P=0.08 ; I^{2}=56 \%$ \\
\hline & Anatomical success at mid-term & 3 & 386 & RR $0.89[0.84,0.96] ; P=0.001$ & $P=0.67 ; I^{2}=0 \%$ \\
\hline \multirow{2}{*}{ Adverse events } & Major complication & 4 & 615 & RR $0.33[0.09,1.28] ; P=0.11$ & $P=0.73 ; I^{2}=0 \%$ \\
\hline & Phlebitis & 4 & 615 & RR $1.39[0.67,2.86] ; P=0.37$ & $P=0.58 ; I^{2}=0 \%$ \\
\hline
\end{tabular}

TABLE 4: Anatomical success and complication rates in each study.

\begin{tabular}{|c|c|c|c|c|c|c|}
\hline \multirow[b]{2}{*}{ Studies } & \multicolumn{2}{|c|}{ Anatomical success rates } & \multicolumn{2}{|r|}{ DVT } & \multicolumn{2}{|c|}{ Sensory disturbances } \\
\hline & MOCA & Thermal ablation & MOCA & Thermal ablation & MOCA & Thermal ablation \\
\hline & \multicolumn{2}{|c|}{ Short-term } & \multicolumn{2}{|c|}{ At the end of study } & \multicolumn{2}{|c|}{ At the end of study } \\
\hline Bootun et al. 2016 [15] & $91.7 \%$ & $91.5 \%$ & $0 \%$ & $1.7 \%$ & $0 \%$ & $0 \%$ \\
\hline Holewijn et al. 2017 [13] & $91.3 \%$ & $99.0 \%$ & $0 \%$ & $0 \%$ & $1.0 \%$ & $2.9 \%$ \\
\hline Lane et al. 2016 [16] & $92.8 \%$ & $91.5 \%$ & $1.2 \%$ & $1.2 \%$ & $0 \%$ & $0 \%$ \\
\hline Vähäaho et al. 2019 [14] & $100.0 \%$ & $100.0 \%$ & $0 \%$ & $0 \%$ & $0 \%$ & $7.6 \%$ \\
\hline Total & $93.4 \%$ & $95.8 \%$ & $0.7 \%$ & $1.4 \%$ & $0.4 \%$ & $3.2 \%$ \\
\hline \multicolumn{7}{|c|}{ Mid-term } \\
\hline Lane et al. 2016 [16] & $86.7 \%$ & $92.7 \%$ & & & & \\
\hline Vähäaho et al. 2019 [14] & $86.4 \%$ & $100.0 \%$ & & & & \\
\hline Holewijn et al. 2017 [13] & $81.6 \%$ & $93.2 \%$ & & & & \\
\hline Total & $84.5 \%$ & $94.8 \%$ & & & & \\
\hline
\end{tabular}

TABLE 5: Adverse events in the included trials.

\begin{tabular}{|c|c|c|c|c|c|c|}
\hline \multirow{2}{*}{ Adverse events } & \multicolumn{2}{|r|}{ Phlebitis } & \multicolumn{2}{|r|}{ DVT } & \multicolumn{2}{|c|}{ Nerve injury } \\
\hline & MOCA & Thermal ablation & MOCA & Thermal ablation & MOCA & Thermal ablation \\
\hline Holewijn et al. 2017 [13] & 12 & 8 & 0 & 0 & 1 & 3 \\
\hline Vähäaho et al. 2019 [14] & 1 & 0 & 0 & 0 & 0 & 5 \\
\hline Bootun et al. 2016 [15] & 0 & 2 & 0 & 1 & Not reported & Not reported \\
\hline Lane et al. 2016 [16] & 3 & 2 & 1 & 1 & 0 & 0 \\
\hline
\end{tabular}

assessor may have contributed to the lower anatomical success rate observed in our study.

The major benefit of MOCA reported in previous reviews was pain intensity reduction during the procedure $[17,18]$. However, we did not perform an analysis of pain intensity because there was a high risk of measurement bias from no participant blinding in the available trials. The potential benefits in reducing the risk of nerve injury might be of considerable clinical importance for MOCA. Our meta-analysis indicated that MOCA had a lower risk of major complications compared with thermal ablation. Nerve injury was seen in $0.4 \%$ and $3.2 \%$ cases after MOCA and thermal ablation, respectively.

There was disparity in terms of anatomical success measure definitions, postprocedure management, and technical aspects of the MOCA procedure. Holejwin et al. reported different results from the others as they used a different sclerosant and categorized DUS image findings into 3 categories instead of 4 [13]. The types of sclerosant used by different studies may have influenced anatomical success. There was substantial heterogeneity in anatomical success at the short-term follow-up in our study. Removing those trials improved the heterogeneity without changing the direction of the effect. Therefore, the heterogeneity in our study might be due to various types of sclerosants and definitions of anatomical success.

There were a few limitations in our meta-analysis. First, we evaluated efficacy only from the anatomical success rate aspect, not from pain reduction, clinical success, or quality of life aspects. Second, there was a wide CI around the effect estimate of adverse event outcomes because there were few events in this meta-analysis. Future trials are required. To 
date, there is one unpublished trial and one ongoing trial investigating the anatomical success of MOCA compared with thermal ablation $[12,19]$. Lastly, the need of data at more than 1 year of outcome measurement at least is suggested.

\section{Conclusion}

Pooled analysis from limited trials demonstrated that MOCA offered fewer major complications (DVT, nerve injury, and skin burns) but lesser anatomical success at the period of more than 6 months but less than 1-year follow-up than thermal ablation. More RCTs together with proper homogeneous data collection are needed to reach optimal information size.

\section{Data Availability}

The data supporting this meta-analysis are from previously reported studies and datasets, which have been cited. The processed data are available in the supplementary files (available here).

\section{Ethical Approval}

The study was approved by the Dr. Soetomo General Hospital Surabaya Ethical Committee in Health Research (1286/KEPK/VII/2019).

\section{Conflicts of Interest}

The authors declare that the research was conducted in the absence of any commercial or financial relationships that could be considered as a potential conflict of interests.

\section{Acknowledgments}

This work did not receive specific funding but was performed as part of Johanes Nugroho employment at the Department of Cardiology and Vascular Medicine, Universitas Airlangga/Dr. Soetomo General Hospital, East Java, Indonesia.

\section{Supplementary Materials}

Forest plot of all outcomes in meta-analysis. (Supplementary Materials)

\section{References}

[1] C. J. Evans, P. L. Allan, A. J. Lee, A. W. Bradbury, C. V. Ruckley, and F. G. Fowkes, "Prevalence of venous reflux in the general population on duplex scanning: the Edinburgh vein study," Journal of Vascular Surgery, vol. 28, no. 5, pp. 767-776, 1998.

[2] G. Marsden, M. Perry, K. Kelley, A. H. Davies, and on behalf of the Guideline Development Group, "Diagnosis and management of varicose veins in the legs: summary of NICE guidance," BMJ, vol. 347, no. 1, article f4279, 2013.

[3] J. I. Almeida, J. Kaufman, O. Göckeritz et al., "Radiofrequency endovenous ClosureFAST versus laser ablation for the treatment of great saphenous reflux: a multicenter, single-blinded, randomized study (RECOVERY study)," Journal of Vascular and Interventional Radiology, vol. 20, no. 6, pp. 752-759, 2009.
[4] A. L. Kerver, A. C. van der Ham, H. P. Theeuwes et al., "The surgical anatomy of the small saphenous vein and adjacent nerves in relation to endovenous thermal ablation," Journal of Vascular Surgery, vol. 56, no. 1, pp. 181-188, 2012.

[5] R. L. Mueller and J. K. Raines, "ClariVein mechanochemical ablation: background and procedural details," Vascular and Endovascular Surgery, vol. 47, no. 3, pp. 195-206, 2013.

[6] R. R. van Eekeren, J. L. Hillebrands, K. van der Sloot, J. P. de Vries, C. J. Zeebregts, and M. M. Reijnen, "Histological observations one year after mechanochemical endovenous ablation of the great saphenous vein," Journal of Endovascular Therapy, vol. 21, no. 3, pp. 429-433, 2014.

[7] M. E. Witte, C. J. Zeebregts, G. J. de Borst, M. M. P. J. Reijnen, and D. Boersma, "Mechanochemical endovenous ablation of saphenous veins using the ClariVein: a systematic review," Phlebology: The Journal of Venous Disease, vol. 32, no. 10, pp. 649-657, 2017.

[8] J. P. T. Higgins, J. A. C. Sterne, J. Savović et al., "A revised tool for assessing risk of bias in randomized trials," Cochrane Database of Systematic Reviews, vol. 10, no. 1, 2016.

[9] R. Ryan and S. Hill, How to GRADE the quality of the evidence Version 3.0, Cochrane Consumers and Communication Group, 2018, July 2019, https://cc.cochrane.org/sites/cc .cochrane.org/files/public/uploads/how_to_grade.pdf.

[10] H. JPT and S. Green, Cochrane handbook for systematic reviews of Interventions Version 5.1.0, The Cochrane Collaboration, 2011, July 2019, https://handbook-5-1.cochrane.org/.

[11] Y. L. Lam, I. M. Toonder, and C. H. Wittens, "Clarivein mechano-chemical ablation an interim analysis of a randomized controlled trial dose-finding study," Phlebology: The Journal of Venous Disease, vol. 31, no. 3, pp. 170-176, 2016.

[12] C. C. Leung, D. Carradice, T. Wallace, and I. C. Chetter, "Endovenous laser ablation versus mechanochemical ablation with ClariVein ${ }^{\oplus}$ in the management of superficial venous insufficiency (LAMA trial): study protocol for a randomised controlled trial," Trials, vol. 17, no. 1, article 421, 2016.

[13] S. Holewijn, R. R. van Eekeren, A. Vahl et al., "Two-year results of a multicenter randomized controlled trial comparing Mechanochemical endovenous Ablation to RADiOfrequeNcy Ablation in the treatment of primary great saphenous vein incompetence (MARADONA trial)," Journal of Vascular Surgery: Venous and Lymphatic Disorders, vol. 7, no. 3, pp. 364-374, 2017.

[14] S. Vähäaho, O. Mahmoud, K. Halmesmäki et al., "Randomized clinical trial of mechanochemical and endovenous thermal ablation of great saphenous varicose veins," The British Journal of Surgery, vol. 106, no. 5, pp. 548-554, 2019.

[15] R. Bootun, T. R. Lane, B. Dharmarajah et al., "Intra-procedural pain score in a randomised controlled trial comparing mechanochemical ablation to radiofrequency ablation: the Multicentre Venefit versus ClariVein for varicose veins trial," Phlebology: The Journal of Venous Disease, vol. 31, no. 1, pp. 61-65, 2016.

[16] T. Lane, R. Bootun, B. Dharmarajah et al., "A multi-centre randomised controlled trial comparing radiofrequency and mechanical occlusion chemically assisted ablation of varicose veins - final results of the Venefit versus Clarivein for varicose veins trial," Phlebology: The Journal of Venous Disease, vol. 32, pp. 89-98, 2016.

[17] J. J. Sun, M. M. Chowdhury, U. Sadat, P. D. Hayes, and T. Y. Tang, "Mechanochemical ablation for treatment of truncal venous insufficiency: a review of the current literature," 
Journal of Vascular and Interventional Radiology, vol. 28, no. 10, pp. 1422-1431, 2017.

[18] D. Boersma, V. N. Kornmann, R. R. van Eekeren et al., “Treatment modalities for small saphenous vein insufficiency: systematic review and metaanalysis," Journal of Endovascular Therapy, vol. 23, no. 1, pp. 199-211, 2016.

[19] D. Boersma, R. R. J. P. van Eekeren, H. J. C. Kelder et al., "Mechanochemical endovenous ablation versus radiofrequency ablation in the treatment of primary small saphenous vein insufficiency (MESSI trial): study protocol for a randomized controlled trial," Trials, vol. 15, no. 1, p. 421, 2014. 